\title{
Bessere Qualität durch neue Gesetze?
}

\author{
Heinz-Michael Just
}

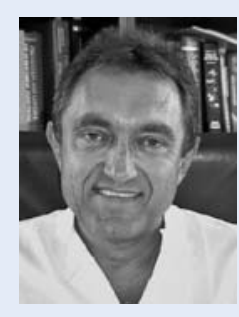

Heinz-Michael Just

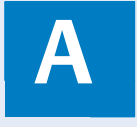

m 4. August 2011 ist das „Gesetz zur Änderung des Infektionsschutzes und weiterer Gesetze“ in Kraft getreten mit dem Anspruch, „die Hygienequalität in Krankenhäusern und bei medizinischen Behandlungen zu verbessern“ (Pressemitteilung Nr. 38 des Bundesministeriums für Gesundheit [BMG]). Die Bundesländer werden deshalb verpflichtet, „bis zum 31. März 2012 Verordnungen zur Infektionshygiene und zur Prävention von resistenten Krankheitserregern in medizinischen Einrichtungen zu erlassen. Diese Verordnungen werden für Krankenhäuser und andere medizinische Einrichtungen gelten." Darunter fallen aber leider nicht die Heime. Warum ist nicht bekannt; man munkelt, wegen der Kosten. Gerade in Heimen bestehen aber erhebliche, oft unbeachtete Risiken. Regelungen zur Infektionsprävention wären deshalb hier besonders wichtig, zumal immer mehr invasive Maßnahmen ambulant durchgeführt werden.

Die Qualität in Sachen Hygiene soll künftig dokumentiert und öffentlich gemacht werden. Die Frage ist nur wie? Grundlage sollen die KRINKO-Empfehlungen sein, bei deren Beachtung „der Stand der medizinischen Wissenschaft“ als eingehalten „vermutet“ wird. Dies bedeutet, dass man auch künftig davon abweichen kann, man muss ggf. nur belegen können, dass man es mindestens gleich gut macht. Die Qualitätsindikatoren sind in Zukunft öffentlich zu machen, damit der Patient sich entscheiden kann, in welches Krankenhaus er geht - oder auch nicht. „Dafür sollen Kriterien zur Messung der Hygienequalität erarbeitet werden, die eine Bewertung und Vergleichbarkeit der Hygienesituation in den Krankenhäusern ermöglichen." Diese gilt es zu finden. Ist die Erfüllung der personellen und strukturellen Voraussetzungen (sinnvoll) so ein Parameter? Oder der Einbau der aktuell von der Industrie beworbenen „antimikrobiellen Technologie“ in Form antimikrobieller Oberflächen, Türgriffe oder - als neueste Errungenschaft - antimikrobielle Betonbausteine (für Nasszellen), deren Nutzen bislang nicht erwiesen ist? Sind es Infektionsraten, die überwiegend unkontrolliert vor Ort erfasst und mangels öffentlich zugänglicher objektiver Daten Manipulationsgefahren ausgesetzt sind? Am ehesten werden es Struktur- und Prozessdaten sein, die aber sichtbar, messbar und transparent darstellbar sein sollten.
Dies beginnt bei der personellen Ausstattung einer medizinischen Einrichtung mit Hygienefachpersonal, um die Sachkunde und damit die Beratungsqualität sicherzustellen. Hierbei werden aber eklatante Versäumnisse der vergangenen Jahrzehnte aufgedeckt, denn es fehlt an ausgebildetem Fachpersonal, insbesondere Krankenhaushygienikern. Für die erforderliche Qualifizierung dieser Personengruppen fehlen bundesweit Ausbildungsstätten und verbindliche, wissenschaftlich fundierte Curricula, die richtige Prioritäten setzen und ein einheitliches Qualitätsniveau garantieren. Die Bundesärztekammer hat eine Fortbildungsregelung für Kliniker zur Qualifikation in Krankenhaushygiene verabschiedet, die den Bedarf kleinerer und mittelgroßer Kliniken analog der KRINKO-Empfehlung decken soll. Der Krankenhaushygieniker klassischer Prägung für Großkliniken bleibt davon unberührt. Aktuell bemühen sich die relevanten Fachgesellschaften, deren Qualifikation speziell in Krankenhaushygiene sicher zu stellen. Um die Weiterbildung von Pflegepersonal zur Hygienefachkraft kümmern sich die Landeskrankenhausgesellschaften, wobei bestehende Curricula diverser Ausbildungsstätten einer dringenden Aktualisierung entsprechend den heutigen Ansprüchen in der Krankenhaushygiene bedürfen.

Ganz neu bietet das BMG eine Förderung von Forschungsprojekten zu „Antibiotikaresistenz, Hygiene und nosokomiale Infektionen“ an, um „geeignete Instrumente und Qualitätsindikatoren zu entwickeln bzw. ... unter Alltagsbedingungen zu validieren“. Hierfür sind 3 Jahre eingeplant, sodass unter Berücksichtigung der Vorlaufzeit mit verwertbaren Ergebnissen frühestens in 4-5 Jahren zu rechnen ist. Wenn dann erst die geforderte Indikatorensuche über eine Expertengruppe des AQUA-Instituts beginnt, vergehen weitere 1 - 2 Jahre, bevor in den Einrichtungen die Qualitätssicherung in der Infektionsprävention Routine werden kann. Sofern die richtigen Parameter beforscht und die Ergebnisse die Eignung bestätigen.

Entscheidend wird auch die öffentliche Diskussion dieser Daten sein und hier können Berufsvertreter, Politiker wie auch die Medien viel zu einer sachlichen Darstellung und objektiven Bewertung beitragen, wenn sie sich ihrer Verantwortung bewusst sind und einer falschen Nutzung durch interessierte Kreise entgegen wirken. Man darf gespannt sein! 